\title{
SURGICAL RECONSTRUCTION OF CHARCOT FOOT NEUROARTHROPATHY, A CASE BASED REVIEW
}

\author{
Tomáš Kučera ${ }^{1,2}$, Pavel Šponer ${ }^{1,2}$, Jaromír Šrot ${ }^{2}$ \\ Charles University in Prague, Faculty of Medicine in Hradec Králové, Czech Republic: Department of Orthopedic Surgery'; \\ University Hospital in Hradec Králové, Czech Republic: Department of Orthopedic Surgery²
}

Summary: Our case-based review focuses on limb salvage through operative management of Charcot neuroarthropathy of the diabetic foot. We describe a case, when a below-knee amputation was considered in a patient with chronic Charcot foot with a rocker-bottom deformity and chronic plantar ulceration. Conservative treatment failed. Targeted antibiotic therapy and operative management (Tendo-Achilles lengthening, resectional arthrodesis of Lisfranc and midtarsal joints, fixation with large-diameter axial screws, and plaster cast) were performed. On the basis of this case, we discuss options and drawbacks of surgical management. Our approach led to healing of the ulcer and correction of the deformity. Two years after surgery, we observed a significant improvement in patient's quality of life. Advanced diagnostic and imaging techniques, a better understanding of the biomechanics and biology of Charcot neuroarthropathy, and suitable osteosynthetic material enables diabetic limb salvage.

Key words: Charcot neuroarthropathy; Diabetic foot; Resectional arthrodesis

\section{Introduction}

Over the past several years, there has been a gradual trend toward increasing efforts for diabetic limb salvage in many countries. The 1-year mortality rate after lower extremity amputation is between $11 \%$ and $41 \%$, and the 5 -year rate is between $39 \%$ and $68 \%$ (1). Diabetic foot ulcers are a major cause of morbidity and possible osteomyelitis. Development of limb-threatening infections may result in a major amputation. Our case report focuses on Charcot neuroarthropathy of the diabetic foot, a progressive condition that results in joint dislocation, pathologic fractures, and severe deformity that often heralds the onset of plantar soft tissue breakdown and ulceration due to plantar dislocation of the tarsal bones and instability of the foot. This impacts patients' quality of life and may represent a significant risk for limb waste. There is no singular cause for the development of the Charcot foot. The current belief is that uncontrolled inflammation leads to osteolysis and is indirectly responsible for the progressive fracture and dislocation. A neurally mediated vascular reflex leading to increased peripheral blood flow and active bone resorption has been proposed as an etiological factor in the development of skeletal destruction in neuropathic patients. Neuropathy is a universal feature of the affected limb (2).

In 1868, Charcot provided a concise description of the neuropathic component of the disease in cases of syphilis (3). In 1936, Jordan was the first to link neuro-osteoarthropathy to diabetes mellitus (4). Midfoot problems are typical, and collapse of the arch creates a rocker-bottom deformity. The most frequent ulcer locations are plantar medial (dislocation of medial cuneiform or navicular bone, and eventually the talus), followed by plantar lateral ulcers (dislocation of cuboid bone) and plantar central ulcers (collapse of the central tarsal-metatarsal region). The optimal treatment protocol remains an issue of debate.

Conservative treatment is the method of choice for many patients, which includes offloading and casting until the swelling has resolved and the temperature of the affected side is within $2{ }^{\circ} \mathrm{C}$ that of the contralateral foot in the acute phase (5). Prescriptive shoes, boots, or weight-bearing braces with frequent monitoring are recommended after an active acute stage to prevent the recurrence of ulceration or the occurrence subsequent deformities (6). However, Saltzman et al. performed a retrospective study of the standard conservative treatment protocol for diabetic feet and concluded that a strict adherence to conservative treatment is linked to poor results and that better management methods are needed (7). In some patients, exostectomy may lead to successful ulcer healing due to symptomatic bony prominences (8-9). Simons et al published the results of operative treatment of patients with Charcot deformity. They corrected the deformity and arthrodesis of tarsal joints without a recurrence of the ulcers (10). Therefore, operative treatment may be superior to conservative management in some cases.

The aim of this study is to present our approach to operative treatment of chronic Charcot neuroarthropathy. 


\section{Case report}

A 65-year-old woman with diabetes mellitus type 1, diagnosed at age 27 years and treated with Humulin Mix 50 12-12-12 IU s.c. daily, and who also had arterial hypertension was referred from the diabetologic out-patient department to our Department of Orthopedic Surgery. She had observed a deformity of her left foot for one and half years and an ulcer in the plantar aspect of the foot for 4 months. The diagnosis of chronic Charcot neuroarthropathy was given (peripheral sensory neuropathy associated with reduced sensation of pain, the classic rocker-bottom deformity with plantar ulceration, corresponding X-ray finding). She was treated conservatively - walking with crutches and non-weight-bearing on the affected limb, orthosis, antibiotics (swab from the ulcer showed Staphylococcus aureus, Acinetobacter species, and Proteus mirabilis, and amoxicillin/clavulanic acid $2 \mathrm{~g}$ daily was recommended due to clinical signs of inflammation and purulent secretion), and regular exchange of the dressing by home care with Curafil Gel (Kendall, USA) and Inadine (Johnson\&Johnson, USA). A noninvasive vascular assessment was performed. Her ankle brachial index was 1.26 , and toe pressure was $98 \mathrm{~mm} \mathrm{Hg}$. Despite good vascular status and complex management, the conservative treatment failed (it lasted seven months): there was insufficient reduction in the size of the ulcer, intermittent exacerbations of infection, full weight-bearing magnified the ulcer and it was impossible to wear a normal footwear. Below-knee amputation was considered.

When we assessed the patient, a typical rocker-bottom deformity was present. In the middle of the sole on the apex of the deformity, there was an ulcer $3 \times 2.3 \mathrm{~cm}$ with granulation tissue. Pulses on the arteria dorsalis pedis and arteria tibialis posterior were palpable. Plantar flexion contracture of the ankle was present. The lag to neutral position was $10^{\circ}$ and plantar flexion was $30^{\circ}$. The skin temperature was nearly equal to that of the right foot (Fig. 1).

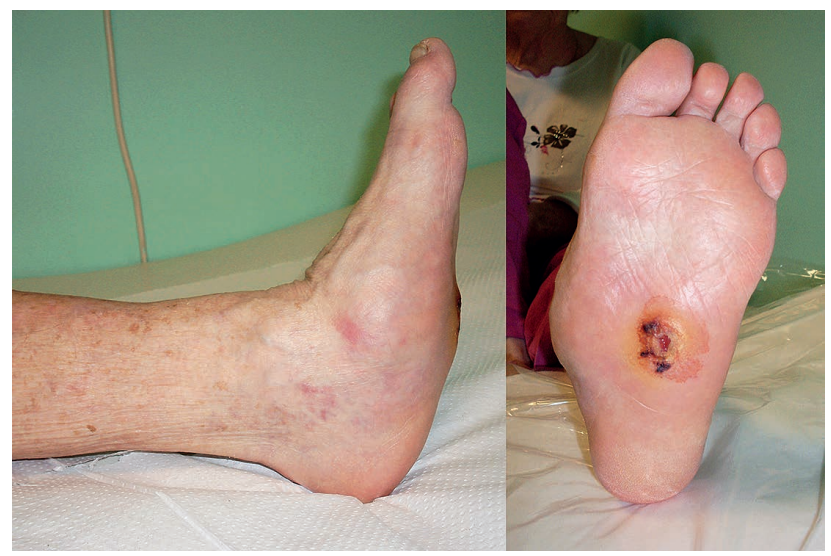

Fig. 1: Clinical appearance of chronic-stage Charcot neuroarthropathy with a rocker-bottom foot deformity. The lag to neutral ankle position of $10^{\circ}$, widening of the foot due to medial and plantar prominences, and plantar ulcer are present.
Lateral and dorsoplantar radiographs with full weight-bearing were performed (Fig. 2).

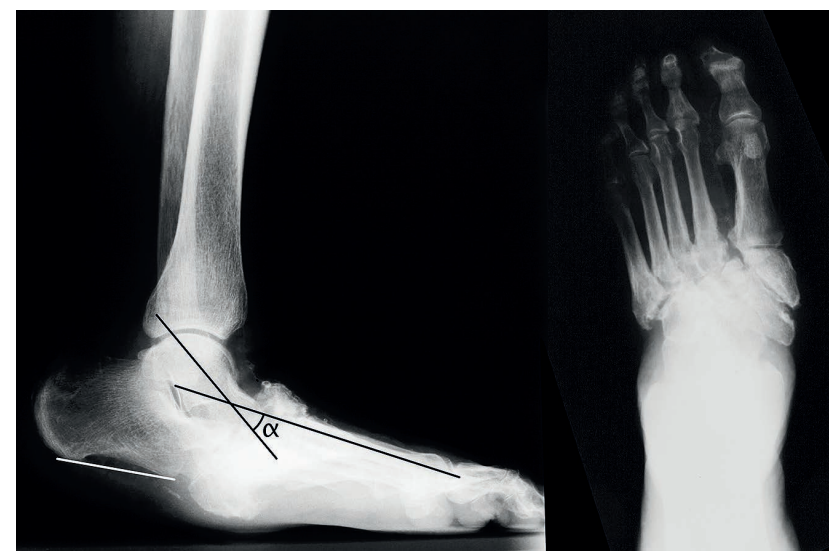

Fig. 2: Preoperative plain radiographs. Lateral view with Meary's angle $34^{\circ}$ (black lines) and negative calcaneal inclination (white line); plantar ejection of the cuboid is visible. Dorsoplantar view with medial prominence of the navicular and medial cuneiform, and abduction of the forefoot in the Lisfranc joint.

Plantar ejection of the cuboid was seen, which caused increased pressure on the sole of the foot and the rise of an ulcer. The ulcer could not heal while the dislocation was present. We measured Meary's angle (lines bisecting the first metatarsal and talus on the lateral radiographic view). We found a negative angle created by a Charcot collapse of $34^{\circ}$. Furthermore, we found a negative calcaneal inclination that corresponded to contracture of the Tendo-Achilles and reduced calcaneus-fifth metatarsal angle. Alignment of the Lisfranc joint was in slight abduction to the forefoot. To better visualize the deformity, we performed computed tomography $(\mathrm{CT})$ scanning with 3-dimensional reconstruction.

Because conservative treatment failed, surgical reconstruction was indicated. We used the Foot and Ankle Ability Measure score (FAAM) and 36-Item Health Survey to compare the preoperative condition with those postoperatively.

The operation was performed under regional anesthesia, without use of a tourniquet. First, the plantar ulcer was excised, and a sample of tissue from the deeper layer was sent for microbiologic assessment. Second, the Tendo-Achilles was percutaneously lengthened using a three-incision technique (medial stab incision $1 \mathrm{~cm}$ proximal to the insertion at the calcaneus, a lateral stab incision $3 \mathrm{~cm}$ proximal to the first one, and another medial stab incision $3 \mathrm{~cm}$ proximal to the middle one; half of the tendon was transected followed by controlled dorsiflexion). Next, both the medial and lateral midtarsal joints were incised to visualize the plantar dislocated navicular and cuboid bone. At first, we performed medially-based osteotomy through the articular surfaces of the Lisfranc joint. The wedge of bone was removed and we manipulated the forefoot into the correct position and held it temporarily with $\mathrm{K}$-wires. Then, the articular surfaces of 
the talus, navicular, and cuneiform bones on the medial aspect, and calcaneus and cuboid bones on the lateral aspect were resected. The bones were moved into anatomic position under fluoroscopic guidance, and the alignment was temporarily held by K-wires. Resected bones were used as autologous bone grafts in talo-navicular fusion. Stable fixation was performed by midfoot fusion bolts. The first bolt was placed in the medial column, entering within the head of the first metatarsal and ending in the talus. The second bolt was placed from the base of the fourth metatarsal through the cuboid into the calcaneus to support the lateral column. Residual bony prominences were removed. Two drains were inserted and suturing was performed. Finally we put a plaster cast over the foot for 3 months. The outcome of the microbiologic assessment from the tissue harvested perioperatively was positive for Staphylococcus species and Propionibacterium species. The patient received intravenous antibiotics for 2 weeks consisting of clindamycin $(600 \mathrm{mg}$ every 6 hours) and ciprofloxacin (400 mg every 12 hours), followed by perorally administered linezolid ( $600 \mathrm{mg}$ every 12 hours) for 2 weeks. The linezolid was chosen because of the lasting secretion from lateral incision and intermediate sensitivity of clindamycin to present bacteria from a new specimen was observed.
Antithrombotic prophylaxis was ensured by enoxaparin (40 mg s.c. daily) for 3 months.

The first postoperative day, we started physiotherapy and non-weight-bearing walking with crutches for home, otherwise the patient used a wheelchair for protection of both legs. There was a complication of delayed healing of the lateral incision ( 3 weeks). We monitored wound through window in plaster.

The patient was followed up in our out-patient department at 6 weeks, when we completely changed the cast and we inspected the skin, next at 12 weeks, 16 weeks, 6 months, 12 months and 24 months after surgery. Twelve weeks after the operation we removed the plaster cast (no oedema, no temperature differential between the two feet), a special frame orthosis was made, and the patient started partial weight-bearing at 16 weeks only with crutches, and full weight-bearing at 6 months after the operation. The orthosis was exchanged for custom-made shoes in 12 months after operation. Twenty four months after surgery, we re-evaluated the patient's FAAM score and 36-Item Health Survey. The FAAM score increased from $41.6 \%$ to $78.6 \%$. Table 1 shows the changes in the 36-Item Health Survey.

The clinical findings and radiography twenty four months after surgery are illustrated in Fig. 3 and 4.

Tab. 1: Thirty six-item health survey: preoperative and postoperative findings.

\begin{tabular}{|c|c|c|}
\hline & Preoperatively & Postoperatively \\
\hline Physical functioning & $20 \%$ & $50 \%$ \\
\hline Role limitations due to physical health & $0 \%$ & $100 \%$ \\
\hline Role limitations due to emotional problems & $0 \%$ & $100 \%$ \\
\hline Energy/fatique & $20 \%$ & $65 \%$ \\
\hline Emotional well-being & $32 \%$ & $76 \%$ \\
\hline Social functioning & $50 \%$ & $87.5 \%$ \\
\hline Pain & $50 \%$ & $100 \%$ \\
\hline General health & $15 \%$ & $50 \%$ \\
\hline
\end{tabular}

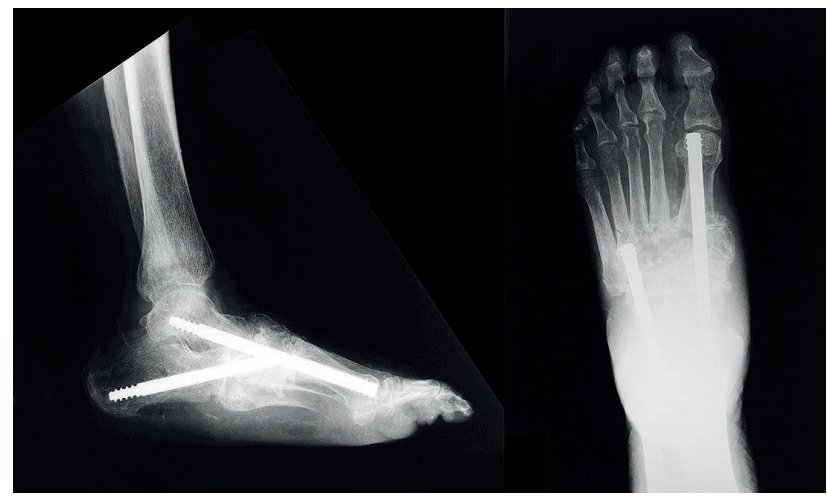

Fig. 3: Postoperative plain radiographs 1 year after surgery. Lateral view with apparent correction of the deformity, normal Meary's angle and positive calcaneal inclination, healed arthrodesis, and two axial screws in place. Dorsoplantar view showing straight position of the forefoot, and healed arthrodesis.

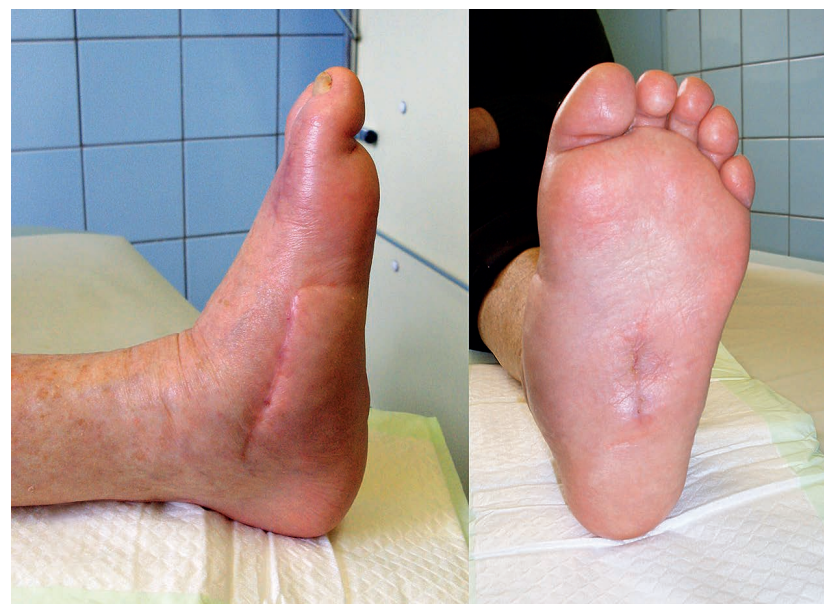

Fig. 4: Clinical appearance after surgical reconstruction, completely healed plantar ulceration, and neutral position of the ankle joint. 


\section{Discussion}

The prevalence of diagnosed Charcot arthropathy in patients with diabetes is reported to be $0.08-7.5 \%$ (11). However, some studies suggest higher prevalence with as many as $13 \%$ of all diabetic patients and $29 \%$ of the neuropathic patients affected (12). Nevertheless, we can assume that due to the Charcot artropathy's clinical resemblance to relatively more common disorders such as osteomyelitis, the diagnosis is often missed or delayed.

In our case, the clinical examination of the contracture of Tendo-Achilles and imaging methods that showed plantar extrusion of bones indicated that conservative treatment would not provide an opportunity for the ulcer to heal. The aim of surgery was to create a stable, plantigrade foot without ulcers that could be accommodated in appropriate shoe gear.

However, the surgical management may have some pitfalls. It is primarily a concern of infectious complications. The presence of neuropathy increases the risk of surgical site infection even in patients without diabetes. Poor longterm glycemic control, and lengthy tourniquet times are also associated with an increased risk of surgical site infection: surgical wound dehiscence with deep infection or pin tract infection in case of external fixator (13-14). Another serious complication might be worsening of vascular status, and also the reconstructing a chronic deformity to a normal alignment could lead to a secondary compromise of blood vessels and ischemia with the necessity of amputation.

Regarding possible severe complications, we insist on a careful preoperative assessment with an emphasis on diabetes compensation, vascular assessment, infection control, and obtaining informed consent from the patient. Cooperation from the patient for the treatment and observing the postoperative recommendations is necessary. The patient's segmental toe pressure was favorable, and the diabetes mellitus was compensated under a diabetologists control. In general, it may be difficult to assess the vascular status in a diabetic foot. We prefer the segmental pressure of the big toe, and angiograms in case of poor results. Ankle-brachial indices may be falsely elevated because of noncompressible vessels, and transcutaneous oxygen tension may be unreliable in cases where the foot is swollen. However, even normal findings do not necessarily mean there has been successful healing. To prevent possible ischemia, we prefer regional anesthesia with a vasodilatation effect, and surgery without use of a tourniquet.

In diabetic foot, managing infection of a chronic wound can be a problem. We often find polymicrobial flora on the surface of an ulcer that does not necessarily correspond to the bacteria in deeper layers. It is a question of appropriate sample harvesting and microbiological assessment. Cultures from the wound must be obtained by removing a small piece of tissue from as deep as possible, which is then sent to the laboratory without delay. In this manner, we can assess not only aerobic but also anaerobic bacteria in the sample. In this case, anaerobic Propionibacterium was present in the deeper layers of the ulcer. In the event of deep infection, the combination of technetium/indium bone scanning or magnetic resonance imaging (MRI) should be considered to rule out the presence of osteomyelitis. However, both diagnostic tools have difficulty differentiating between Charcot foot and osteomyelitis and may be falsely positive in Charcot foot. In case of MRI, differentiation between osteomyelitis and acute or subacute neuroarthropathy requires careful analysis of the location of bone signal alterations, their distribution, and pattern because qualitative changes are often identical. Presence of secondary signs such as adjacent ulcer, cellulitis, and sinus tract is indicative of osteomyelitis (15).

When there is a suspicion of osteomyelitis, we prefer a two-stage procedure. In the first stage, careful debridement and sequestrectomy are followed by the second stage, reconstruction of the foot. The next problem is selecting effective antibiotics during the perioperative period. Appropriate guidelines in the treatment of diabetic foot infection have been published by Infectious Diseases Society of America in 2012 (16). A sample of tissue is harvested during the operation, and the antibiotic therapy is changed immediately, if necessary, when we receive the results of the microbiologic assessment. Possible adverse reactions caused by antibiotic therapy are Clostridium difficile diarrhea. We recommend probiotic supplements.

Resection of osseous plantar prominences in patients with a stable Charcot foot deformity may reduce the pressure on the sole. Such simple exostectomy can be followed with accommodative bracing (17). This less technically-demanding procedure has generally poor results in unstable Charcot foot deformities because the collapse progresses with the recurrence of plantar osseous prominences and ulceration (18).

Our approach to the operative treatment of Charcot neuroarthropathy generally consists of the same principles for all patients; Tendo-Achilles lengthening, resectional arthrodesis of the Lisfranc joint, resectional arthrodesis and realignment of the midtarsal joints, and primary or secondary healing of the eventual soft tissue defect. This approach is based on the idea of superconstructs in the treatment of Charcot foot deformity; that is, extension of arthrodesis to unaffected joints, bone resection for sufficient repositioning without excessive tension on soft tissues, the firmest tolerable implant, and implantation with regard to the best possible mechanical stability (19). Suitable implants are large-diameter axial screws, plantar locking plates, and external fixators. The Ilizarov external fixator frame is useful to compress bone segments to achieve joint fusion, bridge across sites of infected bone, and allow offloading and access to plantar wounds (20). A drawback of external fixation is a pin tract infection making it necessary to remove a wire and the danger of losing the correction. Another disadvantage is the relative expense of the external fixator frame for one patient. The primary advantage of the use of a locking plate along the medial and/or lateral column is the resistance to strain across the fusion site (21). These may be placed medially, laterally, or on the plantar aspect. An additional advantage is 
that locking plates are superior to standard plates in fixation of osteoporotic bones (22). The risk of this technique is the necessity for more extensive soft tissue release for implantation, and a higher risk of infection with exposure of the metal material. In our clinical practice, we prefer large-diameter axial screws. They are placed intramedullary, and the risk of infection is lower than with plates. They overbridge bone destruction and secure the proximal and distal foot segments, and reliably ensure anatomic recreation of Meary's angle. Sammarco et al published a successful application of this technique in Charcot midfoot arthropathy (23). Baravarian Meta-analysis of 154 Charcot foot arthrodeses proved that with precise surgical technique, appropriate postoperative care, and meticulous patient compliance, stability can be restored to the dysfunctional foot (24).

The first step in our reconstruction procedure is Tendo-Achilles lengthening. The prevalence of equinus in the diabetic population has been reported as $10.3 \%$ (25). This deformity plays an important role in elevated plantar pressures, resulting in plantar ulcerations and midfoot breakdown. It is important to evaluate the type of equinus; gastrocnemius or gastro-soleal. In Charcot deformities, we observe gastro-soleal type in most cases. In this condition, we perform either percutaneous three tendon hemisections or, in a severe deformity, Z-plasty lengthening in the frontal plane. The result of this procedure must be a $90^{\circ}$ relationship between the plantar heel and an imaginary line bisecting the fibula. We must avoid tendon over-lengthening. The aim is to re-establish a positive calcaneal inclination angle and to decompress the midfoot. The extent of resectional arthrodesis depends on the type of deformity. We observe the abduction deformity of the forefoot in most cases, thus medially-based osteotomy in the region of Lisfranc joint is performed first.

A comparatively complicated situation is the repair of the midtarsal joints. On the medial side, the navicular is often plantar dislocated, and the talus is nearly vertical and appears to articulate with the medial cuneiform. Similarly on the lateral side, the cuboid is often plantar-dislocated beneath the bases of the fourth and fifth metatarsals. For better exposure, we prefer 3-dimensional CT scanning before surgery, and internal fixation with 2 midfoot fusion bolts medially and laterally. The fixation includes the Lisfranc joint (the first metatarsal medially and the base of the fourth metatarsal laterally). The fixation is completed with application of a plaster cast, which the patient wears for three months. If necessary, we overbridge the midfoot on the medial side with an additional locking plate. However, we assume that the additional metal constitutes a higher risk of infection. When there is a residual soft tissue defect, we prefer negative pressure wound therapy. To enhance healing of the defect, we use split-thickness skin grafts in some cases. During the postoperative course, we prefer long-term non weight-bearing to sustain the healing process of the osteoporotic bone and soft tissues. We must also think of the other leg protection due to possible overloading and the possibility of contralateral Charcot foot.
Two years after surgery, we observed a significant improvement in our patient's FAAM score and in the 36-Item Health Survey. The psychological effect was outstanding; the patient no longer fears the loss of an extremity, and the chronic ulcer has healed. The patient can wear custom-made shoes despite intermittent swelling. The foot was pain free.

Another option might be the measurement of dynamic plantar loading before and after foot reconstruction surgery (26).

\section{Conclusion}

Advanced diagnostic and imaging techniques, a better understanding of the biomechanics and biology of Charcot neuroarthropathy, and suitable osteosynthetic material enables diabetic limb salvage and improves the quality of patients' lives. The pathway to success is long and uncertain, and requires endurance from both the patient and the multidisciplinary healthcare team. Further research and development of our diagnostic and therapeutic methods is essential.

\section{Acknowledgements}

This study was supported by the programme PRVOUK P37/04.

\section{Conflict of interest: none}

\section{References}

1. Frykberg RG. Prevention of lower-extremity amputation in diabetic patients. Diabetic Microvascular Complications Today 2003 May/June: 27-30.

2. Jirkovská A, Kasalický P, Boucek P, Hosová J, Skibová J. Calcaneal ultrasonometry in patients with Charcot osteoarthropathy and its relationship with densitometry in the lumbar spine and femoral neck and with markers of bone turnover. Diabet Med 2001; 18(6): 495-500.

3. Charcot JM. Sur quelques arthropathies qui paraissent dependre d'une lesion du cerveau ou de la moelle epiniere. Arch Physiol Norm et Pathol 1868; 1: 161.

4. Jordan WR. Neuritic manifestations in diabetes mellitus. Arch Intern Med 1936; 57: 307-66.

5. Armstrong DG, Lavery LA. Monitoring healing of acute Charcot's arthropathy with infrared dermal thermometry. J Rehabil Res Dev 1997; 34: 317-21.

6. Rogers LC, Frykberg RG, Armstrong DG, et al. The Charcot foot in diabetes. Diabetes Care 2011; 34: 2123-29.

7. Saltzman CL, Hagy ML, Zimmerman B, Estin M, Cooper R. How effective is intensive nonoperative initial treatment of patients with diabetes and Charcot arthropathy of the feet? Clin Orthop Relat Res 1995; 435: 185-90.

8. Van der Ven A, Chapman CB, Bowker JH. Charcot neuroarthropathy of the foot and ankle. J Am Acad Orthop Surg 2009; 17: 562-71.

9. Brodsky JW, Rouse AM. Exostectomy for symptomatic bony prominences in diabetic Charcot feet. Clin Orthop Relat Res 1993; 296: 21-26.

10. Simon SR, Tejwani SG, Wilson DL, Santner TJ, Denniston NL. Arthrodesis as an early alternative to nonoperative management of Charcot arthropathy of the diabetic foot. J Bone Joint Surg.2000; 82-A: 939-50.

11. Larson SA, Burns PR. The pathogenesis of Charcot neuroarthropathy: current concepts. Diabet Foot Ankle 2012; 3: 122-36.

12. Chisholm KA, Gilchrist JM. The Charcot joint: a modern neurologic perspective. J Clin Neuromusc Dis 2011; 13: 1-13.

13. Wukich DK, Crim BE, Frykberg RG, Rosario BL. Neuropathy and poorly controlled diabetes increase the rate of surgical site infection after foot and ankle surgery. J Bone Joint Surg 2014; 96-A: 832-39.

14. Rogers LC, Bevilacqua NJ, Frykberg RG, Armstrong DG. Predictors of postoperative complications of Ilizarov external ring fixators in the foot and ankle. J Foot Ankle Surg 2007; 46(5): 372-75. 
15. Ledermann HP, Morrison WB. Differential diagnosis of pedal osteomyelitis and diabetic neuroarthropathy: MR Imaging. Semin Musculoskelet Radiol 2005; 9(3): 272-83.

16. Lipsky BA, Berendt AR, Cornia PB, et al. 2012 Infectious Diseases Society of America clinical practice guideline for the diagnosis and treatment of diabetic foot infections. Clin Infect Dis 2012; 54(12): 132-73.

17. Laurinaviciene R, Kirketerp-Moeller K, Hostein PE. Exostectomy for chronic midfoot plantar ulcer in Charcot deformity. J Wound Care 2008; 17: 53-55.

18. Catanzariti AR, Mendicino R, Haverstock B. Ostectomy for diabetic neuroarthropathy of the midfoot. Am J Orthop 2007; 39: 291-300.

19. Sammarco VJ. Superconstructs in the treatment of Charcot foot deformity: plantar plating, locked plating, and axial screw fixation. Foot Ankle Clin 2009; 14 393-407.

20. Pinzur M. Ring fixation in Charcot foot and ankle arthropathy. Tech Foot Ankle Surg 2006; 5(2): 68-73.
21. Sammarco GJ, Conti SF. Surgical treatment of neuroarthropathic foot deformity. Foot Ankle Int 1998; 19: 102-9.

22. Zehnder S, Bledsoe JG, Puryear A. The effects of screw orientation in severely osteoporotic bone: a comparison with locked plating. Clin Biomech (Bristol, Avon) 2009; 24: 589-94.

23. Sammarco VJ, Sammarco GJ, Walker EW jr, Guiao RP. Midtarsal arthrodesis in the treatment of Charcot midfoot arthropathy. J Bone Joint Surg 2009: 91:80-91.

24. Baravarian B, Van Gils CC. Arthrodesis of the Charcot foot and ankle. Clin Podiatr Med Surg 2004; 21(2): 271-89.

25. Lavery LA, Armstrong DG, Boulton AJ. Ankle equinus deformity and its relationship to high plantar pressure in a large population with diabetes mellitus. J Am Podiatr Med Assoc 2002; 92(9): 479-82.

26. Najafi B, Crews RT, Armstrong DG, et al. Can we predict outcome of surgical reconstruction of Charcot neuroarthropathy by dynamic plantar pressure assessment? - A proof of concept study. Gait Posture 2010; 31(1): 87-92.

Received: $26 / 03 / 2014$

Accepted in revised form: 10/09/2014

\section{Corresponding author:}

Tomáš Kučera, M.D., Ph.D., University Hospital, Department of Orthopedic Surgery, Sokolské 581, 50005 Hradec Králové, Czech Republic; e-mail: tomas.kucera@fnhk.cz 